\title{
Hermanos de víctimas de agresiones sexuales: una aproximación a las víctimas indirectas $^{1}$
}

\author{
Siblings of sexually abused victims: An approach to \\ secondary victims
}

\author{
Yanitza Espinoza Angelcos ${ }^{2}$ \\ Carla Förster Merkeß \\ Claudia Capella Sepúlveda
}

Resumen

La presente investigación, de carácter cualitativo, tuvo por objetivo explorar las vivencias de hermanos/as de víctimas de agresiones sexuales en torno a la ocurrencia de la agresión sexual y sus consecuencias. Para esto se realizaron entrevistas a ocho adolescentes hermanos/as de víctimas y a sus adultos a cargo. Los resultados permiten concluir que, los hermanos/as de víctimas de agresiones sexuales pueden ser considerados como víctimas indirectas, en la medida que sufren un malestar subjetivo que es consecuencia de la develación de la situación de agresión sexual. Las características y la profundidad de los efectos negativos sufridos por estos sujetos dependen de factores tales como: la relación con el agresor, el funcionamiento familiar, la relación fraterna y los rasgos del individuo.

Palabras clave: agresión sexual, victima indirecta, develación, vivencia subjetiva.

1 Este artículo es parte de la Memoria para obtener el título de Psicóloga de las dos primeras autoras, la cual se desarrolló bajo la supervisión y dirección de la tercera autora.

2 Psicóloga, Universidad de Chile. E-mail: yanitzaangelcos@gmail.com

3 Psicóloga Universidad de Chile. E-mail: carlaforster@hotmail.com

4 Académica del Departamento de Psicología, Universidad de Chile. E-mail: ccapella@u.uchile.cl 


\begin{abstract}
The purpose of this study was the examination of child sexual abuse victims' siblings experiences, in relation to the sexual abuse and its consequences. The methodology was qualitative, conducting in-depth interviews to 8 adolescents, which were brothers or sisters of victims that were treated in a specialized center, and their adult carer. Conclusions based on the results of the study are that siblings of child sexual abuse victims can be considered as secondary victims, because they suffer as a consequence of the disclosure of the sexual abuse. However, the characteristics and severity of the negative effects suffered will depend on different factors, like: relationship with the offender, family functioning, sibling relationship, and individual characteristics.
\end{abstract}

Key words: sexual abuse, secondary victim, disclosure, subjective experience.

\title{
Planteamiento del problema y perspectiva teórica
}

La agresión sexual ${ }^{5}$ hacia menores de edad corresponde a un tipo de maltrato infantil, constituyendo una de las vulneraciones más graves a los derechos humanos, por lo tanto, se considera víctimas a las personas que son objeto de esta (Martínez, 2000; Neuman, 1989; Selander, 2008).

Definir agresión sexual infantil es una tarea compleja, debido a que los aspectos enfatizados en su conceptualización dependen del ámbito (psicológico, social y legal) en el que esta se inserta. Al respecto, una perspectiva psicosocial proporciona una visión integral del fenómeno y una definición comprensiva del concepto (Canton \& Cortés, 1997; Capella \& Miranda, 2003).

Desde dicha perspectiva, Barudy (1998) plantea como factores estructurales en la génesis de la agresión sexual: la coerción y asimetría de poder entre el adulto y el niño, basadas en la diferencia de edad;

5 Se utilizará el término agresión(es) sexual(es) de forma genérica e indistinta para hacer referencia a las diferentes formas de vulneración de índole sexual. Pese que, el término abuso sexual se utiliza de forma genérica a nivel internacional, este corresponde en la legislación chilena a un tipo específico de delito sexual. 
la vulnerabilidad y la dependencia del niño, lo que impide que este último participe en un verdadero intercambio y decida libremente, transformándose así en un objeto de gratificación sexual para el agresor. De esta manera, el término agresión sexual designa: "el uso abusivo e injusto de la sexualidad. Refleja la idea, además, de que no existe relación sexual apropiada entre un niño y un adulto, atribuyendo la responsabilidad de este tipo de acto exclusivamente al adulto" (Barudy, 1998, p. 161).

Por otra parte, existen diversas clasificaciones de los tipos de agresión sexual infantil, siendo la más significativa aquella que enfatiza el vínculo previo entre la víctima y el agresor. Según lo anterior, una agresión sexual infantil puede ser clasificada en intrafamiliar o extrafamiliar, pudiendo ser el agresor, en esta última, un conocido o un desconocido para el niño y/o su familia (Barudy, 1998; Centro de Asistencia a Víctimas de Atentados Sexuales [CAVAS], 2003).

El concepto de víctima desde una perspectiva psicojurídica, alude al individuo o grupo que padece un daño por acción u omisión, ya sea por responsabilidad propia, ajena o por causa fortuita. Además, es quien sufre un daño por una conducta antisocial, ya sea de responsabilidad propia o ajena, aunque la persona no sea el detentor del derecho vulnerado (Rodríguez, 1990). De esta forma, se considera víctima no solo al que sufre el delito directamente, "sino que se incluye además a los familiares o dependientes inmediatos de la victima directa (VD) y a las personas que hayan sufrido daños al intervenir para asistir a la víctima en peligro o para prevenir la victimización" (VII Congreso de Prevención del Delito y Tratamiento del Delincuente, Milán 1985, citado en Rodríguez, 1990, p. 59). En esta línea, el autor plantea la existencia de una victimización directa y una indirecta, siendo esta última aquella que recae sobre las personas que tienen una relación afectiva estrecha con la víctima directa, produciéndose como consecuencia de la ocurrencia del delito.

Es así que, en casos de agresiones sexuales, la victimización directa recae sobre el sujeto objeto de la agresión, mientras que la victimización indirecta es aquella que sufren los familiares $u$ otras personas cercanas al individuo agredido, quienes se ven afectados por la ocurrencia de la 
agresión sexual en sí misma, su develación y sus consecuencias, al tener que enfrentar el dolor de sus seres queridos y adaptarse a la nueva situación (Echeburúa \& Corral, 2005).

Respecto a lo anterior, se entiende por develación el proceso por el cual una agresión sexual es conocida por personas ajenas a la situación abusiva (Capella, 2010), siendo relevante, al generar en el grupo familiar una reacción que puede adquirir características de crisis, al verse perturbada la homeostasis previa del sistema (Malacrea, 1998).

En relación a las consecuencias generadas a partir de una victimización sexual y su develación, existen variadas investigaciones y literatura asociada al daño sufrido por las víctimas directas (Capella \& Miranda, 2003; Contreras, Capella, Escala, Núneez \& Vergara, 2005; Echeburúa \& Corral, 2005; Finkelhor \& Browne, 1985; Núñez, 2010, entre otros). Por el contrario, son escasas las investigaciones o la literatura asociada a la repercusión que tendría la victimización sexual a nivel familiar, las que se enfocan principalmente en los padres, indagando en las reacciones de estos ante la develación y en los efectos conductuales y emocionales que sufrirían (Abarza \& Olivares, 2000; Barudy, 1998; Hooper, 1992; Malacrea, 1998; Mannarino \& Cohen, 1996; Mc Court, Peel \& O Carroll, 1998; Perrone \& Nannini, 1997). En esta línea, Arriagada y Thiers (2005) señalan que a largo plazo dichos efectos pueden conllevar a la aparición de sintomatología, llegando incluso a adquirir características postraumáticas. $\mathrm{Al}$ respecto, se describen en los padres de niños/as que han sido víctimas de agresiones sexuales principalmente sentimientos de culpa, pérdida de confianza, aislamiento, temor, vergüenza, rabia y pérdida de control, entre otros.

En cuanto a las consecuencias de la agresión sexual en otros miembros de la familia, se aprecia que las investigaciones se limitan a plantear un posible perjuicio, sin profundizar al respecto (Barudy, 1998; Malacrea, 1998, entre otros).

Debido a esto, se realizó una búsqueda exhaustiva utilizando los principales conceptos (tanto en inglés como en español) asociados a esta investigación (abuso sexual, víctimas indirectas, hermanos; sexual abuse, indirect victims, secondary victims, siblings, brothers) en diversas bases 
de datos (EBSCO, Elsevier, SCIELO, Medline, Proquest, entre otras). También se buscaron estos conceptos en las principales revistas vinculadas a la temática de las agresiones sexuales (child abuse and neglect, journal of child sexual abuse, child abuse review, aggression and violent behavior, entre otras), y en los catálogos de diversas universidades chilenas (UCH, PUC, UDP, UFRO, etc.). Junto con esto, se realizó consultas a expertos nacionales respecto a bibliografía disponible en el tema.

Luego de esta búsqueda, se encontraron solo tres artículos a nivel internacional y ninguno a nivel nacional, que abordan directamente la temática, los cuales explicitan que se han realizado pocos estudios sobre los efectos del abuso sexual en los hermanos/as de las víctimas, siendo escasa la literatura al respecto (Baker, Tanis \& Rice, 2002; Hill, 2003; Tavkar \& Hansen, 2011). Incluso Tavkar y Hansen (2011) plantean la necesidad de la realización de más estudios que identifiquen el impacto del abuso sexual en los hermanos/as de las víctimas, señalando que no se le ha dado la importancia que merece, lo cual es necesario para el desarrollo de intervenciones apropiadas.

En los estudios sobre los hermanos/as de víctimas (Baker et al., 2002; Hill, 2003; Tavkar \& Hansen, 2011) se plantean como principales repercusiones asociadas a la agresión sexual y a su develación en estos sujetos la presencia de sentimientos de tristeza, vergüenza y estigmatización; preocupación por la víctima directa; culpa por no disminuir o detener la agresión sexual, o sentir que debieran proteger al hermano. Por otra parte, pueden experimentar conflictos de lealtades con el agresor, y presentar confusión y ambivalencia de sentimientos en torno a este, especialmente cuando es la figura parental. Muchas veces el agresor es alejado de la familia, lo cual puede asociarse a sentimientos de pérdida o enojo hacia la víctima. A su vez, puede presentarse confusión debido a la falta de información respecto al tema, y sentimientos de enojo y frustración por no haberse enterado antes o haberse enterado de manera indirecta acerca de la situación, o rabia con los padres debido a que muchas veces la información en la familia es entregada de manera parcial, o se establece como secreto. Por otro lado, los hermanos se ven expuestos al estrés familiar que esta situación causa, sintiendo amenazado el sentido de 
seguridad acerca de las relaciones al interior de la familia, y pueden presentar esfuerzos por no expresar sentimientos negativos asociados a la develación y a sus consecuencias. En general, los hermanos no víctimas reciben escaso apoyo de los padres y de los profesionales involucrados, que focalizan sus energías en la víctima directa. Todo esto puede generar conflictos, resentimientos y envidias con el hermano agredido, e incluso se puede culpar a este de la agresión y sus consecuencias (Baker et al., 2002; Bentovin, 1991, en Baker et al., 2002; Hill, 2003; Tavkar \& Hansen, 2011). Junto con esto, otros estudios vinculados tangencialmente al tema, plantean dificultades en la percepción de los miembros de la familia y sus relaciones, en hermanos de niños víctimas de agresiones sexuales (Lipovsky, Saunders \& Hanson, 1993; Meyer, 1983).

Es por esto que los estudios destacan la necesidad de tratamiento de las familias de los niños víctimas de agresiones sexuales, y específicamente de los hermanos/as (Baker et al., 2002; Tavkar \& Hansen, 2011).

En base a lo expuesto, la presente investigación se orienta a explorar las vivencias de los hermanos/as de víctimas de agresiones sexuales, a partir de las percepciones que estos tienen de la ocurrencia de la trasgresión sexual y sus consecuencias, considerando que los hermanos de una víctima directa podrían constituirse como víctimas indirectas.

Es importante destacar que este estudio surge como una propuesta desde el Centro de Asistencia a Víctimas de Atentados Sexuales (CAVAS), en donde los profesionales del equipo clínico reparatorio han pesquisado la necesidad de los hermanos de las víctimas directas de recibir apoyo psicosocial, asociado a la repercusión que habría tenido en ellos la develación y las consecuencias familiares de la agresión sexual. En este sentido, el presente estudio responde a una necesidad e inquietud que surge desde la práctica clínica con víctimas. Por otra parte, a partir de la revisión bibliográfica realizada, de acuerdo a nuestro conocimiento, se podría plantear que sería la primera investigación a nivel nacional que busca aproximarse a las vivencias de los hermanos de las víctimas de agresiones sexuales, y en este sentido, constituye un aporte tanto práctico como teórico en el ámbito psicojurídico, en su área de acción victimológica, en la medida que entrega elementos relevantes para la elaboración de programas de reparación. 
Es así como este estudio permite visualizar las experiencias y necesidades de los hermanos de las víctimas directas, favoreciendo una comprensión más integral del fenómeno. Por otra parte, posibilita el pesquisar la necesidad o no de intervención y apoyo que podrían requerir tales sujetos.

A partir de lo anterior, resulta relevante explorar y describir las vivencias de hermanos de víctimas de agresiones sexuales en relación a la ocurrencia de la agresión sexual y sus efectos. Específicamente se pretende: indagar en la posible influencia que tiene la agresión sexual y sus consecuencias sobre la relación entre la víctima directa y sus hermanos; conocer la forma en que los hermanos de las víctimas directas perciben al agresor a partir de la develación; conocer el modo en que las consecuencias familiares repercuten en los hermanos de las víctimas directas; indagar en las consecuencias sufridas por los hermanos de las víctimas directas, a partir de la trasgresión sexual; y explorar la necesidad de intervención terapéutica en hermanos de víctimas de agresiones sexuales.

\section{Metodología}

Para llevar a cabo el presente estudio se escogió un enfoque cualitativo, al ser este el más pertinente cuando existe escasa información respecto del objeto a investigar. Dicho enfoque, presenta la ventaja de favorecer el acceso a los procesos subjetivos y a los puntos de vista de los propios actores sociales (Flick, 2004; Krause, 1995; Rodríguez, Gil \& García, 1999). Además, esta investigación se identifica con la fenomenología, para esta perspectiva los discursos son producto del modo con que las personas definen su mundo, por ende, su misión es develar el proceso de interpretación que llevan a cabo las personas (Taylor \& Bogdan, 1998).

Este estudio es de tipo exploratorio-descriptivo, ya que se aborda un tema escasamente investigado, además se busca describir y especificar las características del fenómeno a estudiar. Finalmente, esta investigación es de corte transversal, puesto que la información fue recogida en un momento determinado para obtener los datos (Hernández, Fernández \& Baptista, 2006; Sampieri, 2006). 
Para efectos de esta investigación, el universo muestral está conformado por adolescentes hermanos de víctimas de agresión sexual, menores de edad, que acuden al Centro de Asistencia a Víctimas de Atentados Sexuales de la Región Metropolitana (CAVAS).

Los participantes del estudio fueron escogidos mediante muestreo teórico, es decir, la elección de los sujetos fue guiada, tanto por la posibilidad de adquirir nueva información, teniendo en consideración el material ya obtenido y el conocimiento emanado de este, como por características y rasgos considerados relevantes en la revisión teórica realizada y que tienen directa relación al problema planteado (Flick, 2004). Con el fin de identificar los posibles sujetos a incluir en la investigación, se realizó un catastro en CAVAS por medio de un sondeo del número de hermanos de cada víctima atendida en el Centro y su caracterización, a través de la revisión de fichas clínicas y de libros de ingreso de pacientes a dicha institución ${ }^{6}$.

Se establecieron como criterios de inclusión y exclusión los siguientes: ser hermano(a) de un menor de edad víctima de agresión sexual atendido en el CAVAS; tener entre 12 y 18 años de edad; no haber sido objeto de una agresión sexual o que existiesen sospechas al respecto; que el agresor fuera un conocido o familiar tanto de la víctima directa como de su familia nuclear.

Además, se incluyó dentro de la muestra a los adultos responsables de cada uno de los adolescentes participantes del estudio, con el fin de obtener mayores antecedentes sobre las vivencias asociadas a la agresión sexual y recabar aquellos aspectos observables (sintomatología) no advertidos por los participantes, que pudiesen complementar la información obtenida.

Finalmente, la muestra quedó conformada por 8 adolescentes ${ }^{7}$, de entre 13 y 18 años de edad. De estos, tres son hermanos de víctimas de agresión sexual por parte de un conocido y los otros cinco son hermanos de víctimas de agresión sexual intrafamiliar (ver Tabla No1).

\footnotetext{
6 Se consideraron los casos vigentes en CAVAS durante el transcurso de la investigación, la que tuvo lugar entre el segundo semestre del ańo 2010 y los inicios del 2011.

7 Pese a que la muestra se conforma por ocho adolescentes, fueron entrevistados solo siete de estos, ya que uno en el transcurso del proceso decidió no continuar participando, siendo posible entrevistar solamente a su madre, cuyo relato fue incluido dentro del análisis.
} 


\section{Tabla 1}

Caracterización de los participantes de la muestra

Hermano de víctima directa

(VI)
Víctima directa de agresión sexual

(VD)

\begin{tabular}{|c|c|c|c|c|c|c|}
\hline 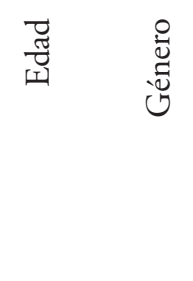 & 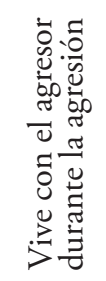 & 胥 & 离 & 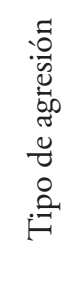 & 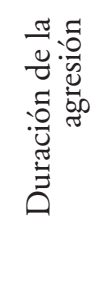 & 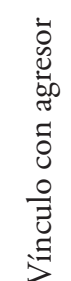 \\
\hline
\end{tabular}

A 17 Femenino Sí 13 Masculino Violación $\begin{gathered}\text { Episodios } \\ \text { reiterados }\end{gathered} \begin{gathered}\text { Hijo de desora del Sí } \\ \text { hogar }\end{gathered}$

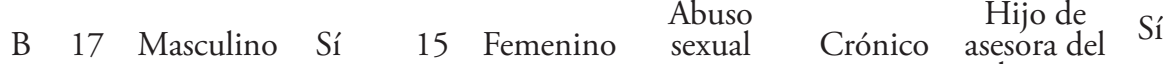
hogar

C 16 Masculino No 14 Femenino $\begin{aligned} & \text { Abuso } \\ & \text { sexua }\end{aligned}$

Pareja de Sí mayor

Abuso Episodio Pareja de Sí

D 17 Femenino Sí 10 Femenino sexual $\begin{gathered}\text { Episodio único } \\ \text { la madre }\end{gathered}$ $\begin{array}{llll}\text { Abuso } & \text { Episodios } & \text { Paterno } & \text { Sí } \\ \text { reiterados }\end{array}$

E 14 Masculino Sí 12 Femenino sexual

Paterno

$\begin{array}{llll}\text { Abuso } & \text { Episodios } & \text { Fraterno } & \text { Sí } \\ \text { sexual } & \text { reiterados }\end{array}$.

F 18 Femenino Sí 9 Femenino sexua

$\begin{array}{llll}\text { Abuso } & \text { Episodios } & \text { Fraterno } & \text { Sí } \\ \text { sexual } & \text { reiterados }\end{array}$ Frato

G 13 Masculino Sí 9 Femenino

Crónico $\begin{gathered}\text { Primo } \\ \text { hermano }\end{gathered}$

H 13 Femenino No 15 Femenino

Abuso

No 
Para acceder al discurso de los sujetos participantes, respecto de sus vivencias asociadas a la agresión sexual sufrida por sus hermanos, se escogió como técnica de recogida de datos la entrevista en profundidad, la que permite obtener información acerca de un fenómeno específico, del cual se elabora un listado de ejes temáticos que buscan focalizar la entrevista (Rodríguez et al., 1999).

Es así como se construyeron dos pautas de entrevista, una para los adolescentes participantes y otra para los adultos a cargo, las cuales se basaron en una revisión de la literatura especializada en victimología y fenomenología de las agresiones sexuales, sobre las consecuencias en la víctima directa y sus figuras significativas. Dichas pautas fueron revisadas por un experto en la temática y elaboradas a partir de los siguientes ejes temáticos: vivencias asociadas a la develación de la agresión sexual en el hermano de la víctima directa; consecuencias de la agresión sexual para el entorno familiar; efectos de las consecuencias familiares en el hermano no víctima de agresión sexual; consecuencias de la agresión sexual para el hermano de la víctima directa: impacto emocional, cambios en su rutina, cambios en su salud (presencia de sintomatología); relación entre víctima directa y su hermano no agredido sexualmente, previo y posterior a la develación; percepción de la figura del agresor, previo y posterior a la develación; impresiones y valoración de la necesidad de atención psicológica especializada en agresiones sexuales.

Cabe señalar que, la metodología de análisis utilizada en el presente estudio está basada en los ejes de la teoría fundamentada, la cual es llevada a cabo a través de las estrategias de muestreo teórico y de un método comparativo constante. Este último, permite codificar y analizar de manera simultánea los datos para desarrollar conceptos. Mediante la comparación continua de los datos recabados, los conceptos son depurados, se identifican sus propiedades, se exploran sus interrelaciones y se los integra en una teoría coherente (Trinidad, Carrero \& Soriano, 2006).

Una vez transcritas las entrevistas, se procedió a analizar la información obtenida mediante la técnica de análisis de contenido. Para esto, se desglosó la información de las entrevistas en citas (frases y párrafos) 
que corresponden a las unidades de análisis, que fueron categorizadas a través de conceptos que representan su contenido. Luego las categorías emanadas, fueron agrupadas en categorías centrales, dando importancia a aquellos contenidos que muestran determinadas tendencias o puntos de vista diferentes de lo mismo (García, Ibáńez \& Alvira, 1994; Mayer \& Quellet, 1991 en Gómez, 2000; Pérez, 2000).

De las unidades, emergieron diversas categorías en torno a: las vivencias de los participantes asociadas a la ocurrencia y consecuencias de la agresión sexual; la calidad de las relaciones entre los involucrados, directa e indirectamente en la agresión sexual; el posible daño de los hermanos de las víctimas de agresiones sexuales; entre otras.

\section{Consideraciones éticas}

Otros elementos estimados relevantes en la conformación de la muestra son los aspectos éticos, planteándose así el requerimiento de que los entrevistados estuviesen informados de la ocurrencia de la agresión sexual. Además, se consideró la apreciación de los psicólogos tratantes de la víctima directa, excluyendo casos en que las entrevistas podrían constituir un factor estresor en aquellas familias que presentaran un significativo nivel de inestabilidad. Finalmente, se tomó en consideración la voluntariedad de participación tanto del adolescente como del adulto a cargo.

Con el objetivo de indagar respecto a los criterios de selección y éticos, se recurrió a los terapeutas y asistentes sociales encargados de cada caso (víctima directa). Es así que, el primer contacto con los posibles participantes fue realizado por los psicólogos, quienes consultaron a sus pacientes si estaban de acuerdo con que se invitara a sus hermanos y figuras responsables a participar en el estudio, explicándoles a grandes rasgos en qué consistía este. Una vez otorgada la autorización de las víctimas directas, los terapeutas tomaron contacto con los adultos a cargo y luego con los posibles jóvenes participantes, invitándolos a formar parte del presente estudio. 
Aquellas personas que accedieron a participar fueron contactadas por las investigadoras. En una primera instancia se citó a cada adolescente junto a su respectivo adulto a cargo, indistintamente de la edad del joven (12-18 años) y se les explicó en qué consistía la investigación mediante la lectura de un consentimiento informado, el que debía ser firmado por los dos, ya que se consideró la voluntariedad e interés de participación de ambos como requisito. En una primera instancia, se realizó la entrevista con el adulto y, en una segunda, se llevó a cabo con el adolescente participante.

Cabe destacar que, se ofreció una devolución (de carácter voluntario) sobre apreciaciones clínicas respecto a la victimización indirecta (asociada a los eventos sexualmente abusivos), llevándose a cabo cinco devoluciones con los adolescentes y sus respectivos adultos a cargo (estas no fueron consideradas como parte del análisis).

\section{Resultados}

A continuación se presentan los resultados más significativos que emergen del análisis de las entrevistas. La información recogida a partir del discurso de los participantes se desglosa en citas que dan lugar a conceptos relevantes que son agrupados en categorías para la comprensión del fenómeno. De este modo, se incluirán viñetas con citas textuales de los adolescentes entrevistados para ejemplificar los análisis expuestos.

Los resultados fueron generalmente coincidentes entre la percepción de los adolescentes y sus adultos a cargo, sin embargo se priorizará los análisis desde el discurso de los mismos adolescentes, y cuando hayan diferencias con respecto a la percepción de los adultos responsables, estas serán explicitadas.

\section{Influencia de la agresión sexual y sus consecuencias sobre la relación fraterna}

Respecto de este punto surge como relevante que el comportamiento reactivo de la víctima directa a la agresión sexual influyó de manera negativa en la relación entre los hermanos. Generalmente, 
este comportamiento reactivo se presentó durante la ocurrencia de los hechos sexualmente abusivos, aun cuando los adolescentes no tuviesen conocimiento de tales hechos:

"Empezó a cambiar de actitud (VD) y peleaba harto. Yo le decía algo y él me llevaba la contraria (...) hubo un tiempo en que se puso súper pesado también” (entrevistado A).

Sin embargo, los participantes dieron cuenta de un cambio positivo en la relación fraterna, tras la develación, lo que podría asociarse tanto a que la víctima directa desplegaría conductas más adecuadas al entorno (por la interrupción de la agresión sexual y por la percepción de apoyo familiar), como por la asunción de un rol más comprensivo por parte del hermano.

En esta línea, fue posible apreciar que los adolescentes entrevistados logran percibir un daño asociado a la agresión sexual en la víctima directa a nivel de la personalidad, la relación con los otros, el comportamiento, la imagen del sí mismo, entre otros. Refiriendo además, la presencia de sintomatología reactiva a los hechos sexualmente abusivos y a la develación. Lo anterior, da cuenta de esfuerzos por parte de los hermanos por comprender el sufrimiento de la víctima, lo que refleja la presencia de sentimientos de empatía hacia esta última:

"Yo cuando escucho eso, en ese momento tenía ganas de abrazarla y estar todo el día con ella (...) y después la aprendi a entender, a entender un poco más a decir bueno: pero porque ella era así conmigo antes, no es que ella sea asi" (entrevistado B).

En este punto, se observa una percepción distinta en los adultos responsables de los adolescentes, que reportan la percepción de un cambio negativo en la relación fraterna tras la develación, que se asocia a las consecuencias de la develación y a la tensión generada al interior de la familia:

"Hubo un desgaste de ellos como hermanos" (madre de A).

Percepción acerca de la figura del agresor tras la develación

$\mathrm{Al}$ respecto, la mayor parte de los sentimientos de los adolescentes entrevistados hacia el agresor se hallan mediados por la calidad de la 
relación previamente establecida con dicho sujeto, aun cuando hay algunos sentimientos comunes. En este sentido, se aprecia que, tras la develación, la totalidad de los hermanos de las víctimas directas dieron cuenta de sentimientos de rabia hacia el agresor.

En cuanto a los elementos diferenciales, cuando hay una relación significativa previa a la develación entre el agresor y el hermano no victimizado, surgen sentimientos ambivalentes hacia esta figura, caracterizados por una fluctuación de emociones que transitan desde afectos negativos hasta la necesidad de rescatar aspectos positivos de dicho individuo:

"Es una sensación, me duele decirlo (...) de repente echarlo de menos, porque hay, habia como un apego (...) pero cuando sé a quien estoy echando de menos, me da rabia, por todo lo que ha pasado (...) ahora pa' mi hay como un desprecio ante él, lo odio en realidad, pero a pesar de todo eso, igual se echa de menos" (entrevistado B).

A diferencia de lo anterior, cuando no hay un vínculo afectivo estrecho con el agresor, no se aprecia ambivalencia en los adolescentes, sino que el principal sentimiento que emerge es la rabia, que se traduciría en deseos de venganza y confrontación. Junto con ello se pesquisó un sentimiento de temor hacia el agresor, asociado a una percepción omnipotente de dicha figura, en los casos en que la personalidad de este sujeto y las características propias de la agresión eran consideradas como violentas. Esta percepción omnipotente se refleja en la idea de no contar con los recursos suficientes para resguardarse y proteger al resto de la familia de los posibles daños que pudiera causar el agresor:

"Yo le dije: mamá tengo miedo, puede venir, no sé, me da miedo que le pueda hacer algo a $Z(V D)$, sabe en qué colegio va, sabe donde está" (entrevistado A).

Repercusión de las consecuencias familiares de la agresión sexual en los hermanos de las victimas directas

Respecto de las consecuencias familiares generadas por la agresión sexual, emana del discurso de los participantes el reconocimiento de esta 
como un perjuicio que involucra a la totalidad del sistema familiar, que da lugar a cambios que afectarían a sus diversos miembros (como individuos, subsistemas y sistema), llegando a ser significada la develación como un antes y un después en la historia familiar.

Uno de estos cambios tiene relación con el rol que desempeña el hermano de la víctima directa en el grupo familiar, de modo tal que cuando estos sujetos son mayores a la víctima directa y han cumplido una función parentalizada ${ }^{8}$ previamente, tras la develación se exacerba su rol protector hacia la víctima directa. Otro de estos cambios surge a partir de la reconfiguración de jerarquías y relaciones familiares, en donde los padres delegarían responsabilidades de cuidado al hermano mayor no victimizado, dando lugar a la asunción de un rol adultizado ${ }^{9}$ por parte de este último.

Sin embargo, a partir del discurso de las figuras responsables, se aprecia que aquellas víctimas indirectas que son menor edad $(\mathrm{G}, \mathrm{H})$, manejan escasa información respecto a la agresión sexual, debido a que sus padres los excluirían intencionalmente de la entrega de esta información, aludiendo a la falta de recursos y de madurez que estas tendrían para integrarla:

"Me enteré por mi mamá, ella me contó (...) me llamó a su pieza y ahi me lo dijo (¿y qué te dijo, te acuerdas?) No exactamente, no sé lo que me dijo" (entrevistado $\mathrm{H}$ ).

"Nunca esto se ha conversado en la mesa: oye esto pasó, jno, porque ella (VI) tiene 13 años!, la quisimos tener más fuera de esto, fuera de darle una preocupación (...) nosotros no le contamos nada (...) no se ha profundizado el tema con ella para no inmiscuirla”(padres de H).

Por otra parte, los adolescentes entrevistados dan cuenta de la percepción de un trato diferencial por parte de sus padres a favor de la víctima directa y en desmedro de ellos, en la forma de prestar más atención y cuidados al hijo víctima, quedando en segundo plano las necesidades del resto de los hijos. Este trato diferencial genera en los hermanos no victimizados emociones de rabia y disgusto, que aparecen en el discurso

8 Parentalización, es la asignación del rol parental a uno o más hijos de un sistema familiar o la asunción de ese rol por parte del hijo. Implica un modo de inversión de roles que está relacionado con una perturbación de las fronteras generacionales.

9 Adultización, asunción de un rol adulto por parte de un niño o adolescente, asumiendo responsabilidades inadecuadas para su etapa del desarrollo, al no encontrarse preparados psicológicamente (cognitiva ni emocionalmente) para ellas. 
como una crítica al estilo de crianza. Junto a ello, se aprecia que los hermanos participantes visualizan la presencia de beneficios otorgados a la víctima directa por su condición de víctima, lo que es interpretado por ellos como una manipulación de las relaciones, que refleja la percepción de una ganancia secundaria, ya que a partir de un evento negativo (agresión sexual) surgen aspectos que favorecen a dicha persona:

"No es excusa, si la Y(VD) viene a responder muy mal, para mi no es excusa que abuso o no abuso, ella puede controlarlo, yo creo" (entrevistado B).

Consecuencias sufridas por los hermanos de las víctimas, a partir de la trasgresión sexual

La develación implica para la totalidad de los sujetos evaluados un evento disruptivo que genera una repercusión emocional de alta magnitud en ellos, siendo las reacciones más frecuentes un impacto emocional y la incredulidad inicial ante los hechos develados. Respecto del impacto emocional, un elemento recurrente es la dificultad de significar la experiencia en un primer momento y la consecuente expresión de emociones negativas. Por otra parte, la incredulidad inicial se da ante la dificultad de integrar la idea de que un ser querido pueda dañar a tal magnitud a una persona afectivamente significativa. Debido a esto los sujetos tienden, en un primer momento, a negar la ocurrencia de los hechos sexualmente abusivos. Es así como la develación da lugar a una tensión y ansiedad para la familia y sus integrantes, incluyendo a los hermanos de la víctima.

Los participantes reportan diversos sentimientos asociados a la ocurrencia de la agresión sexual. En primer lugar, se aprecia la presencia de sentimientos de culpa y autorreproches asociados a no poder impedir la ocurrencia o prolongación de la agresión sexual por no tener conocimiento de esta, ni haberse dado cuenta. Estos sentimientos se originarían a partir de diferentes elementos, tales como: el rol que cumplen los hermanos mayores de las víctimas directas en el grupo familiar y la vinculación afectiva que estas tendrían con los protagonistas de la situación sexualmente abusiva:

"Como que yo nunca noté nada, entonces también me dio rabia (...) senti que el trabajo que me habian pedido que yo hiciera no lo hice 
bien. Mi mamá me dijo que yo cuidara a mis hermanos y me dije: 'yo no los cuidé bien, no les presté suficiente atención'"(entrevistado A).

En segundo lugar, aparecen sentimientos de traición asociados al vínculo y a la confianza, en la medida que una figura con quien se mantenía una relación afectivamente estrecha transgrede la confianza que se le otorgó:

"E (VI) se desilusionó mucho, de todo lo que él (agresor) le habia enseñado, valores y cosas y después saber esto, como que se le derrumbó el papá que él valoraba tanto" (madre de E).

En tercer lugar, surgen sentimientos asociados al temor que adquiriría las siguientes formas: sentimientos de indefensión vinculados a la percepción de no contar con recursos para hacer frente a la amenaza externa, que surge como inminente e imposible de enfrentar, dando lugar a una imagen debilitada de sí mismo, afectando su autoimagen y percepción de eficacia; desconfianza generalizada asociada a la percepción que los otros podrían configurar una fuente de peligro y dañar al formarse un vínculo cercano con estos; y percepción de mundo amenazante que corresponde a una visión del entorno como hostil y como fuente de peligro constante, que daría lugar a un temor permanente a ser dañado al establecer contacto con el medio:

"Después de lo que le pasó, ni en mi papá confio, en nadie confio (...) yo ya no salgo pa' afuera, desde lo que le pasó a la T (VD) (...) le tomé miedo de que me fuera a pasar a mi también" (entrevistado F).

Finalmente, surgen sentimientos de pérdida hacia la figura del agresor, debido al distanciamiento y a la ausencia de una persona con quien se mantenía una vinculación afectiva significativa, pese a que su alejamiento es entendido como una consecuencia de los actos perjudiciales que habría cometido hacia el grupo familiar:

"Me acuerdo y digo: 'ojalá no hubiese pasado nunca eso, ojalá nunca él', yo lo consideraba mi hermano, ojalá estuviese en la casa" (entrevistado B).

En relación a los efectos de la develación en sí mismos, los hermanos de las víctimas perciben un cambio en su persona, a nivel 
de pensamiento y/o su rutina. Además, dan cuenta de la aparición de sintomatología de diversa expresión (recuerdos intrusivos, perjuicio en el rendimiento, aislamiento, desregulación emocional, ánimo depresivo, llegando en algunos casos a pensamientos de muerte) tras tomar conocimiento de la agresión sexual:

"Yo en ese momento estaba mal, reaccionaba mal, si me decian cosas yo reaccionaba mal, contestaba a gritos (...) por ejemplo, peleaba mucho con mi familia y salia y era como súper loca, como que no me interesaba nada" (entrevistado D).

"Me hizo cambiar el pensamiento de ese momento que era como de niño, como más joven (...) todas las experiencias que pasan, como que igual cambian el pensamiento de uno (...) como que uno madura" (entrevistado C).

Es importante destacar que los hermanos de las víctimas directas reportaron postergar su propio sufrimiento en función de apoyar al resto de la familia. Esto hace alusión a una acción consciente de situar en segundo plano el propio malestar causado por la develación, por considerarlo en ocasiones menor al de la víctima directa y al de los padres:

"Mi mamá estaba peor que yo, porque ella sentía mayor culpa que la que yo tenía, pero yo dije: no puedo, no puedo estar yo mal, porque mi mamá ya está mal y $Z(V D)$ está mal (...) entonces yo tampoco me iba a deprimir más" (entrevistado A).

Por otra parte, los adolescentes entrevistados en su mayoría atribuyen la responsabilidad de los hechos sexualmente abusivos al agresor. No obstante, se pudo apreciar, a partir del contenido latente de los discursos de los hermanos entrevistados, que estos asignaban cierta responsabilidad a la víctima directa, en la medida que hacían referencias a características personales de esta última, que la habrían hecho objeto de victimización, o bien que la responsabilizan implícitamente por haber develado tardíamente:

"Mi mamá me preguntaba: 'oye iy él no abusaba de ti, no te tocaba?' y ese tipo de cosas, o sea, yo no soy como la Y (VD). Si asi fuera, yo no me llevaría tan bien con él y no habría pasado, no hubiese sido asi todo esto" (entrevistado B). 
Respecto a lo anterior, una de las madres entrevistadas plantea el supuesto que su hijo culpa a la víctima directa por las consecuencias familiares asociadas a la develación:

"A lo mejor si le puede echar la culpa a la U(VD) de todo lo que pasó (...) porque de repente cuando el chico habla del papá, el E (VI) mira a la U y le dice: si estuviera el papá aquí, qué estariamos haciendo" (madre de E).

Por otra parte, se observó en los entrevistados la necesidad de buscar explicaciones acerca de la causa de la agresión sexual y sus consecuencias, por medio de hipótesis que explicasen, por una parte, el comportamiento del agresor y, por otra parte, el comportamiento reactivo a la agresión de la víctima directa.

Necesidad de intervención terapéutica en hermanos de víctimas de agresiones sexuales

En relación a este punto, fue posible observar durante las entrevistas que aquellos sujetos que habían cursado un proceso terapéutico asociado a la victimización sexual de su hermano (A, B, D), eran capaces de relatar en forma clara e integrada sus vivencias vinculadas a la agresión sexual y sus consecuencias, lo que daría cuenta de cierto grado de elaboración del evento disruptivo. Por el contrario, aquellos sujetos que no contaron con apoyo psicológico, presentaron durante la entrevista dificultades significativas para expresar sus vivencias de forma coherente, tendiendo al desborde emocional, sin embargo, explicitaron que el contexto de entrevista fue valorado positivamente como un espacio de escucha.

\section{Conclusiones y discusión}

En primer lugar, los hermanos de las víctimas de agresiones sexuales pueden ser considerados como víctimas indirectas, ya que experimentan subjetivamente un dolor o malestar vinculados a la victimización sexual y sus consecuencias (Echeburúa \& Corral, 2005). Al respecto, la develación 
para estos sujetos es un evento disruptivo que les genera un fuerte impacto emocional, que con el paso del tiempo podría dar lugar a sentimientos de culpa, rabia, traición, temor, desconfianza e indefensión, así como a sintomatología, incluyendo cambios conductuales. Esto es coincidente con otros estudios respecto a los hermanos/as de víctimas de agresión sexual (Baker et al, 2002; Hill, 2003; Tavkar \& Hansen, 2011).

En segundo lugar, fue posible pesquisar consecuencias similares en los hermanos víctimas indirectas y las descritas en la literatura respecto a la víctima directa (Capella \& Miranda, 2003; Contreras, Capella, Escala, Núñez \& Vergara, 2005; Echeburúa \& Corral, 2005; Finkelhor \& Browne, 1985; Núñez, 2010, entre otros). De este modo, los efectos de la agresión sexual en esta última coinciden con los efectos de la develación en las víctimas indirectas que se expresan tanto a nivel conductual como emocional. Por otra parte, las vivencias de los hermanos de las víctimas directas asociadas a la develación cursarían en dos fases, de forma similar al modo en que se presenta el daño psicológico en las víctimas directas, surgiendo en un primer momento una reacción de sobrecogimiento, seguido de pensamientos de incredulidad y pobreza de reacción y, en un segundo momento, vivencias afectivas de mayor intensidad tales como: dolor, rabia, impotencia, culpa y miedo.

Asimismo, coinciden algunos factores mediadores del daño psicológico presente en las víctimas directas con aquellos que median el malestar subjetivo experimentado por los hermanos víctimas indirectas (Capella $\&$ Miranda, 2003; Contreras, Capella, Escala, Núñez \& Vergara, 2005; Echeburúa \& Corral, 2005; Finkelhor \& Browne, 1985; Núñez, 2010, entre otros). Entre estos factores se encuentran: la relación previa con el agresor, las características familiares, las características personales del sujeto -que incluye el nivel de vulnerabilidad y los recursos psicológicos de afrontamiento disponibles-, las pérdidas sufridas y el apoyo recibido por otros integrantes de la familia. Por otra parte, no se apreciaron diferencias en relación al género ni a la cronicidad de la agresión sexual, y algunas diferencias menores en cuanto a la edad de la víctima indirecta y su orden dentro de la fratría.

Un aspecto relevante en la conformación de las vivencias de los hermanos víctimas indirectas asociadas a la agresión sexual es la calidad de la 
relación establecida previamente entre los sujetos mencionados y el agresor. Cuando ha existido una relación estrecha entre estos, la reacción ante la develación cursa en tres etapas, al igual que en las figuras parentales según la literatura (Mc Court, Peel \& O'Carrol, 1998), a saber, pensamientos de incredulidad seguidos de aceptación de la ocurrencia de los hechos que da lugar a un fuerte impacto emocional. De igual modo, la calidad de la relación entre la figura del agresor y el hermano de la víctima directa influye en los sentimientos que surgen hacia esta figura tras la develación, sentimientos que coinciden con los experimentados por la víctima directa. $\mathrm{Al}$ respecto, cuando la relación previa es estrecha aparecen sentimientos ambivalentes y de pérdida, culpa y rabia, por el contrario, cuando hay ausencia de una vinculación cercana tienen lugar sentimientos de rabia y una percepción omnipotente del agresor.

En relación a esto último, la visión omnipotente del agresor puede resultar en sentimientos de indefensión que llevarían a una generalización de la amenaza externa y a la consiguiente percepción de mundo amenazante. Esto generaría un cambio en el sistema de creencias, reflejado en la suposición de un mundo injusto, que lleva al sujeto a resguardarse del medio a través de conductas agresivas y/o aislamiento, dificultando el establecimiento de relaciones profundas y el vincularse con otros. Lo anterior afectaría el desarrollo integral del individuo, sobre todo en la etapa de la adolescencia, en donde la relación con los pares es fundamental para la conformación de la identidad.

Un elemento central que emerge en este estudio son las consecuencias que atañen de manera específica a los hermanos víctimas indirectas, las cuales se hallan vinculadas a las repercusiones de la agresión sexual a nivel familiar. Al respecto, la develación genera estrés y cambios a nivel familiar, los que adquieren la forma de una rigidización y/o intercambios de los roles preexistentes, de modo tal que los hermanos víctimas indirectas (que son mayores a la víctima directa) adoptan funciones parentales, asumiendo así responsabilidades inadecuadas para su etapa de desarrollo. En algunos casos, los adolescentes pueden llegar a postergar su propio sufrimiento para ser un soporte emocional para sus padres. 
Otro aspecto específico de las vivencias de los hermanos, tiene relación con la percepción de ser tratados por los padres de manera diferente a la víctima directa, lo que daría cuenta de una posible rivalidad entre los hermanos. En relación a lo anterior, fue posible apreciar en las entrevistas que los padres presentaban dificultades para centrar sus discursos en el hermano víctima indirecta, refiriéndose en la mayoría de los casos a sus propias vivencias o a la víctima directa, lo que se constituye en un factor de riesgo, al ser visualizado el daño en los hermanos solo de manera parcial. Algunos de estos elementos son coincidentes con los resultados de otros estudios acerca de las repercusiones en los hermanos/as de víctimas de agresiones sexuales (Baker et al, 2002; Hill, 2003; Tavkar \& Hansen, 2011), y otros resultan novedosos respecto a estos estudios.

A partir de lo expuesto es posible concluir que la expresión del daño en los hermanos víctimas indirectas es variable, viéndose influida por: la configuración previa a nivel individual, familiar y social; las particularidades de las vivencias asociadas a la agresión sexual y develación; y los factores mediadores. Además, se puede señalar que su expresión a nivel comportamental posee características inespecíficas, ya que las conductas desplegadas (sintomatología) podrían ser originadas a partir de diversos eventos vitales negativos, haciendo difícil para el entorno de estos sujetos detectar el daño vinculado a una victimización indirecta. Sin embargo, las vivencias subjetivas son específicas a la develación, permitiendo asociar esta a la respuesta psicoafectiva de los hermanos de las víctimas directas.

Pese a que los resultados de este estudio proporcionan información relevante para la comprensión de las vivencias subjetivas de los hermanos de víctimas de agresiones sexuales, se tornan visibles ciertas limitantes, entre ellas se puede mencionar: las escasas investigaciones en el tema específico de los hermanos de las víctimas de agresión sexual, lo que impidió contar con un referente más preciso en torno al foco de investigación, por lo que la aproximación al grupo de interés respondió a parámetros genéricos surgidos de estudios previos asociados a las víctimas directas o bien a sus figuras parentales; así como la necesidad de establecer criterios éticos que redujeron el número de participantes. 
Teniendo en consideración lo expuesto en el presente estudio, se estima necesario continuar realizando investigaciones que involucren las vivencias de niños y jóvenes asociadas a la victimización indirecta en agresiones sexuales, puesto que esta investigación es una primera aproximación a un fenómeno escasamente estudiado y que implica una gran complejidad, al existir múltiples variables involucradas (individuales, familiares, sociales, entre otras). Es así que, se torna relevante realizar estudios que incluyan a niños menores de 13 años, cuyas vivencias podrían dar cuenta de otras variables mediadoras, otras manifestaciones de daño, y conflictos asociados a la ocurrencia de la agresión sexual y sus consecuencias.

Los resultados obtenidos en este estudio dan cuenta de la necesidad de apoyo psicosocial que requieren los hermanos de las víctimas de agresiones sexuales, en la medida que estos se ven afectados por su ocurrencia, develación y consecuencias para la víctima directa y los otros integrantes de la familia. En esta misma línea, Baker et al. (2002) destacan que, en general, los hermanos/as de víctimas de agresiones sexuales no son incluidos en el tratamiento, sin embargo, destacan la necesidad de incorporarlos. La importancia de incluirlos en el tratamiento radica en poder acoger el impacto que esta experiencia causa en ellos, así como en prevenir que sean victimizados, en tanto se ha estudiado que estarían en un mayor riesgo. Además, Tavkar y Hansen (2011), destacan la importancia del apoyo de los hermanos/as para la recuperación de los niños/as que han sido víctimas directas. En esta línea, una posible intervención beneficiosa podría ser el apoyo grupal para los hermanos (Baker et al., 2002; Tavkar \& Hansen, 2011).

De este modo, se propone que la participación de los hermanos víctimas indirectas en un proceso de terapia psicológica permitiría que puedan elaborar sus propias vivencias, lo que además beneficiaría a las víctimas directas y a sus figuras cuidadoras, en la medida que todo el sistema familiar es afectado. Es así que la realización de investigaciones que propongan modelos de intervención, ya sea individual o grupal, implicarían un aporte significativo tanto en el ámbito teórico como de la práctica psicojurídica, más específicamente en el área de acción victimológica en su vertiente reparatoria. 


\section{Referencias}

Abarza, P. \& Olivares, A. (2000). Estudio exploratorio descriptivo de interacciones del sistema familiar en familias con abuso sexual infantil. Tesis para optar al título de Psicólogo, no publicada. Universidad de Chile.

Arriagada, M. \& Thiers, M. (2005). Diseño, Implementación y Validación de una Intervención Psicoeducativa Grupal para Padres no Agresores de Niños Victima de Abuso Sexual. Memoria para optar al título de Psicólogo, no publicada. Universidad de Chile.

Baker, J., Tanis, H. \& Rice, J. (2002). Including siblings in the treatment of child sexual abuse. Journal of child sexual abuse, 10(3), 1-16.

Barudy, J. (1998). El dolor invisible de la infancia: Una lectura ecosistémica del maltrato infantil. Barcelona: Paidós.

Canton, J. \& Cortés, M. (1997). Malos tratos y abuso sexual infantil. Barcelona: Siglo Veintiuno Editores.

Capella, C. (2010). Develación del abuso sexual en niños y adolescentes: Un artículo de revisión. Revista Chilena de Psiquiatría y Neurología de la infancia y adolescencia, 21(1), 44-56.

Capella, C. \& Miranda, J. (2003). Diseño, implementación y evaluación piloto de una intervención psicoterapéutica grupal para niñas víctimas de abuso sexual. Memoria para optar al título de Psicólogo, no publicada. Universidad de Chile.

Centro de Asistencia a Víctimas de Atentados Sexuales [CAVAS] (2003). Centro de Asistencia a Victimas de Atentados Sexuales, CAVAS Metropolitano: 16 años de Experiencia. Santiago: Instituto de Criminología, Policía de Investigaciones de Chile.

Contreras, L. Capella, C. Escala, C. Núñez, L. \& Vergara, P. (2005). Evaluación de daño psíquico en casos de agresiones sexuales a menores. Cuadernos de Psicología Jurídica. No 2. Santiago de Chile.

Echeburúa, E. \& Corral, P. (2005). Cómo evaluar las lesiones psíquicas y las secuelas emocionales en las víctimas de delitos violentos. Psicopatología clínica, legal y forense, 5, 57-73.

Finkelhor D. \& Browne A. (1985). The Traumatic Impact of child sexual abuse. A conceptualization. American Journal of Orthopsychiatry, 55(4), 530-541.

Flick, U. (2004). Introducción a la investigación cualitativa. Madrid: Ediciones Morata, S.L.

García, M., Ibáñez, J. \& Alvira, F. (1994). El análisis de la realidad social: métodos y técnicas de investigación. Madrid: Alianza Editorial. 
Gómez, M. (2000). Análisis de contenido cualitativo y cuantitativo: Definición, clasificación y metodología. Revista de Ciencias Humanas - UTP, 20. Universidad Tecnológica de Pereira, Colombia. Extraído el 15 de noviembre de 2010 desde: http://www.utp.edu. $\mathrm{co} /$ chumanas/revistas/revistas/rev20/gomez.htm

Hernández, S., Fernández, C \& Baptista, P. (2006). Metodología de la Investigación. México: Editorial McGraw-Hill.

Hill, A. (2003). Issues facing brothers of sexually abused children: implications for professional practice. Child and Family Social Work 2003, 8, 281-290.

Hooper, C. (1992). Madres sobrevivientes al abuso sexual de sus niños. Buenos Aires: Editorial Paidós.

Krause, M. (1995). La investigación cualitativa: un campo de posibilidades y desafíos. Revista Temas de Educación, 50, 19-39.

Lipovsky, J., Saunders, B. \& Hanson, R. (1993). Parent-Child Relationships of Victims and Siblings in Incest Families. Journal of Child Sexual Abuse, 1(4), 35-50.

Malacrea, M. (1998). Trauma y Reparación: El tratamiento del abuso sexual en la infancia. Barcelona: Editorial Paidós.

Mannarino, A. \& Cohen, J. (1996). Family-Related Variables and Psychological Symptom Formation in Sexually Abused Girls. Journal of Child Sexual Abuse, 5(1), 105-120.

Martínez, J. (2000). Prevención del abuso sexual infantil: Análisis crítico de los Programas Educativos. Revista Psykhe, 9(2), 63-74.

Mc Court, J., Peel, J. \& O'Carroll, P. (1998). The effects of Child sexual abuse on the protecting parent(s): Identifying a Counselling Response for secondary victims. Counselling Psychology Quarterly, 11(3), 283-299.

Meyer, S. (1983). Family perceptions of abused children and their siblings. Child abuse \& neglect, 7(1), 107-115.

Neuman, E. (1989). Victimología: El rol de la Victima en los delitos convencionales y no convencionales. Buenos Aires: Editorial Universitaria.

Núńez, L. (2010). Evaluación de daño psíquico en niños preescolares que han sido víctimas de agresión sexual a partir del test de apercepción infantil $C A T-A$. Tesis para optar al grado de Magíster en Psicología, mención Psicología Clínica Infanto-Juvenil. No publicada. Universidad de Chile.

Pérez, G. (2000). Investigación cualitativa: retos e interrogantes II. Técnicas y análisis de datos. Madrid: Editorial La Muralla. 
Perrone, R. \& Nannini, M. (1997). Violencia y abusos sexuales en la familia: Un abordaje sistémico y comunicacional. Buenos Aires: Editorial Paidós.

Rodríguez, L. (1990). Victimología. Estudio de la víctima. Segunda edición. México D.F.: Editorial Porrúa.

Rodríguez, G., Gil, G. \& García, E. (1999). Metodología de la investigación cualitativa. Málaga: Ediciones Aljibe.

Sampieri, R. (2006). Metodología de la investigación. México D.F: Editorial McGraw Hill.

Selander, M. (2008). Género, Maltrato e Infancia: Manual dirigido a los Equipos de proyectos especializados en maltrato y abuso sexual grave de la red de SENAME. Realizado por la Asociación Chilena Pro Naciones Unidas (ACHNU).

Tavkar, P. \& Hansen, D. (2011). Interventions for families victimized by child sexual abuse: Clinical issues and approaches for child advocacy center-based services. Aggression and Violent Behavior, 16(3), 188199.

Taylor, S.J. \& Bogdan, R. (1998). Introducción a los métodos cualitativos de investigación. Barcelona: Editorial Paidós.

Trinidad, A., Carrero, V. \& Soriano, R. (2006). Teoría fundamentada "Grounded Theory": la construcción de la teoría a través del análisis interpersonal. Madrid: Centro de Investigaciones Sociológicas.

Fecha de recepción: 01 de agosto de 2011.

Fecha de aceptación: 14 de diciembre de 2011. 